\title{
Note technique au sujet de l'éboulis englacé de Laux Montaux (Baronnies, Drôme)
}

Monsieur Luc Descroix

\section{Résumé}

Résumé : On décrit un éboulis englacé situé dans les Baronnies. Sa localisation, relativement basse en latitude et altitude dans les Préalpes Françaises, en fait un phénomène rare et méritant une étude approfondie. Il a été le siège d'un glissement-coulée en 1978, probablement lié à la présence de la glace.

\begin{abstract}
Abstract: The ice-pit scree ofLaux Mentaux (French Southern Alps, France). This note is about an iced scree in the Baronnies massif. Its location, at relatively low both altitude and latitude makes it a rare phenomenon. So it seems necessary to achieve thorough investigations on this site. In the same place a landslide-mud flow occurred in 1978, probably linked with the presence of ice.
\end{abstract}

\section{Citer ce document / Cite this document :}

Descroix Luc. Note technique au sujet de l'éboulis englacé de Laux Montaux (Baronnies, Drôme). In: Revue de géographie alpine, tome 89, $n^{\circ} 3,2001$. pp. 90-94;

doi : 10.3406/rga.2001.3052

http://www.persee.fr/doc/rga_0035-1121_2001_num_89_3_3052

Document généré le 06/06/2016 


\section{Note technique au sujet de l'éboulis englacé de Laux Montaux (Baronnies, Drôme)}

\section{Luc Descroix}

LTHE-IRD, BP 53, 38041 Grenoble Cedex 9

descroix@hmg.inpg.fr

Une crête du massif préalpin des Baronnies comporte sur son flan Nord un éboulis dont on a signalé précédemment qu'il devait être englacé (Descroix, 1985), eu égard à une série de témoignages et d'observations réalisées sur le terrain. Etant donné que cet englacement probable commence à être connu (un panneau "Le Glacier " a même été installé en 1998 sur l'emplacement de la carrière qui a permis de mettre en évidence cette particularité), il serait souhaitable que des recherche soient réalisées sur ce phénomène rare à cette altitude et cetre latitude.

\section{Historique}

Les habitants de ce secteur des Baronnies connaissent depuis toujours l'existence de cette particularité, les témoignages des habitants de Laux Montaux, Chauvac, Villeboisles-Pins et d'autres villages plus lointains sont unanimes dans ce sens-là.

Les observations permettant de soupçonner un " microclimat " avaient trait :

- au jaunissement précoce des arbres à la fin de l'été ;

- à la présence de verglas plus fréquents que ce que la seule position du site dans le versant laissait attendre ;

- à la température observée sur place en été, bien plus fraîche qu'aux alentours ;

De plus, la lavande vraie (lavandula officinalis), cultivée ou spontanée, y vient très bien, et on sait que la lavande vraie aime les endroits les plus frais en ubac.
En 1976, lors de l'ouverture (réouverture?) de la carrière, le conducteur de l'engin de travaux publics utilisé a été contraint, en plein été, d'arrêter l'exploitation (sauvage ?) très vite, du fait de la présence de la glace, qui gênait considérablement l'extraction de matériaux. On a $\mathrm{vu}$, à cette occasion, et lors d'autres extractions de matériaux postérieures, des blocs de glace mêlés aux éboulis, et ce en plein été.

\section{Observations}

En février 1978, après une longue période neigeuse, et en même temps que d'autres phénomènes du même type dans la même région, un effondrement-coulée s'est produit juste en aval de ce site (figures 1 et 2). Celuici est composé d'une partie amont qui correspond à l'écroulement d'un pan de versant, peut-être lić à un dégel suivant cette abondante chute de neige, ayant pu entrainer une mise en charge d'une parrie du versant par les eaux d'infiltration. Au bas du versant, les matériaux arrachés lors de l'écroulement ont été simultanément pris en coulée, laquelle a fossilisé le fond du vallon adjacent sur plus de 300 mètres de longueur. Le cours d'eau émissaire a dû ré-entailler un nouveau lit, sur la droite (coté Nord) de la coulée, pour remplacer l'ancien lit recouvert de 4 ou 5 mètres d'alluvions par endroits. Depuis cette datelà, l'ensemble n'a pas sensiblement évolué, la couléc est reconquise par la végétation et n’a plus rebougé.

Régulièrement, des relevés de température sont effectués, en plein été et dans l'aprèsmidi (quand la température sous abri est de 


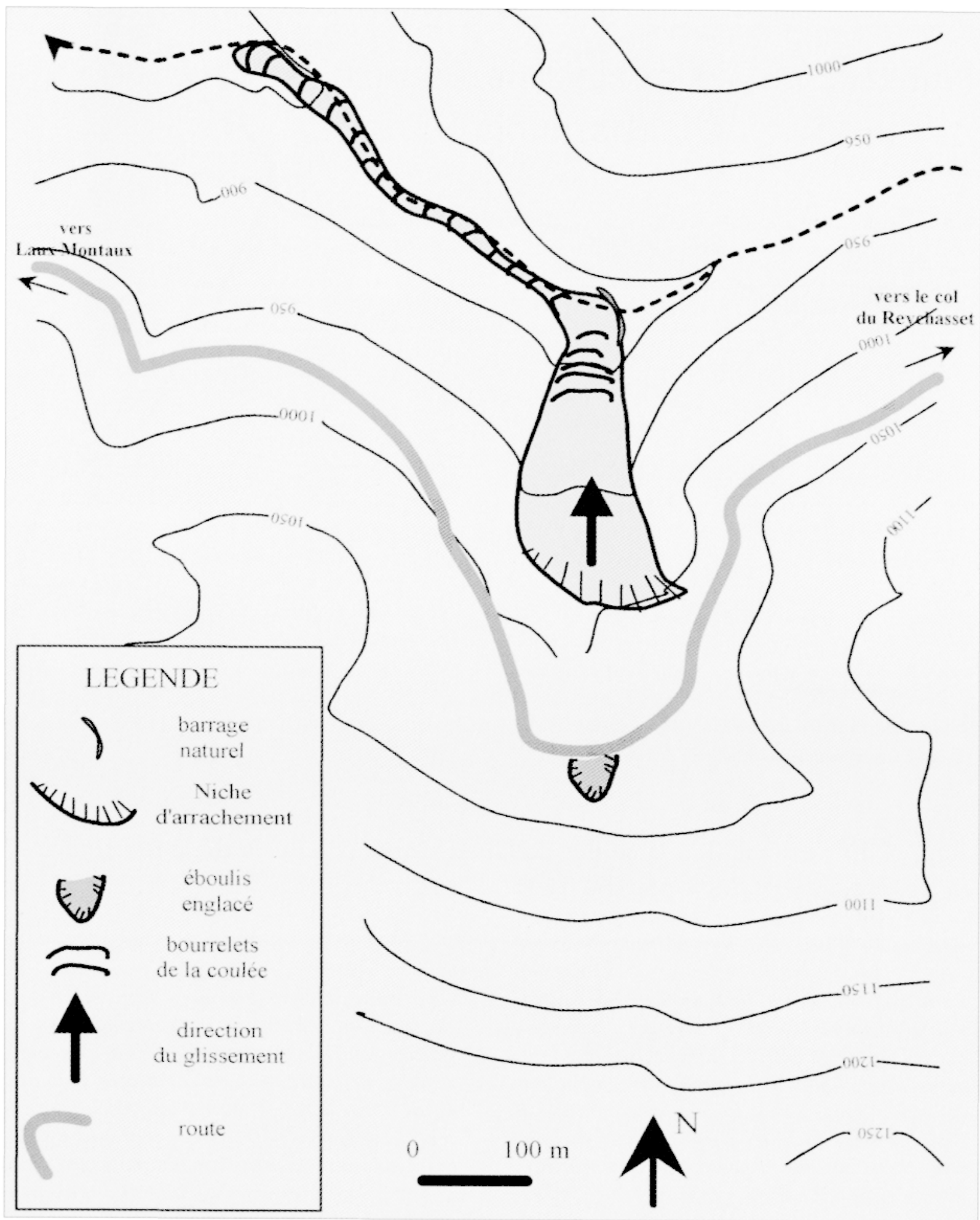

Figure 1 : La coulée boueuse de Laux-Montaux, plan

plus de $25^{\circ}($ ), et l'on mesure a chaque fois des valeurs comprises entre 0 et $1^{\circ}(;$ en glissant un thermometre entre les cailloux (a 10 ou $20 \mathrm{~cm}$ de profondeur), au fond de la carriere a présent abandonnce.
Glacière ou éboulis englacé : une étude en cours par Yannick Robert (thésard I( iA) pourait apporter des connaissances sur ce phénomìne. 


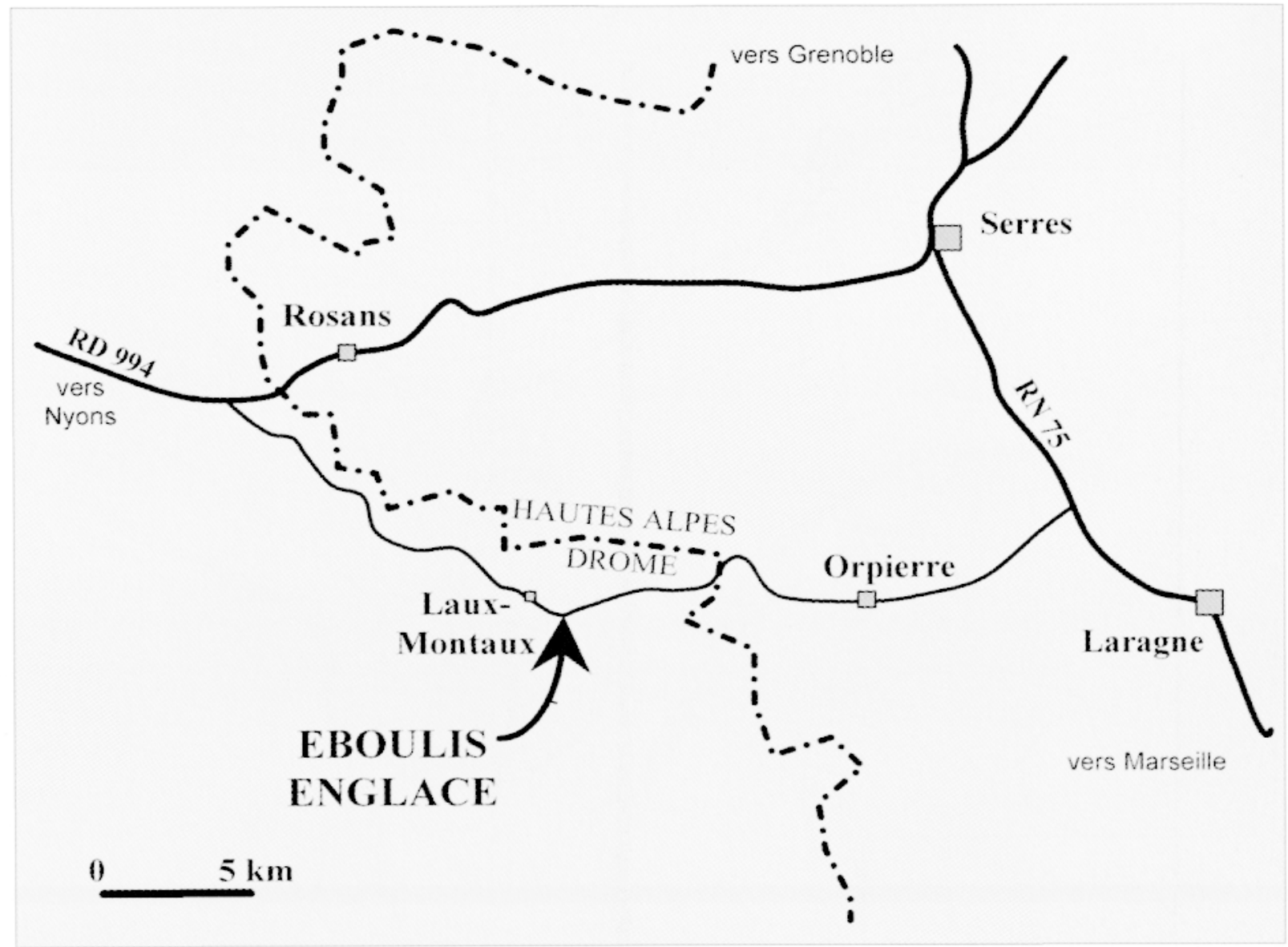

Figure 2 : Localisation du " glacier » de Laux-Montaux

\section{Rappel du texte relatif à la coulée de Laux-Montaux dans la thèse de III' cycle de Luc Descroix (1985, pp. 164-173)}

"La coulée bouense de laux-Montanx.

Cétte coulée a eu lieu sur le territoire de la commune de Laux-Montaux (I)rome); au-dessus du village, en février 1978 (soit quelques jours aurant le glissement de Saint-(yrice et à peu près en méme temps que celui de Montmorin - il sagit de deux localités baronniardes -), apres une longue période neigeuse qui concerna lé région à cetue période. Cet affaissement a affecté essentiellement l'aboulis "stabilisé" qui est superposé à toute une série d'affleurements rocheux alternativement mameax ou calcaires : de bas en haut du versant : 200 mètres de marno-calcaire, $140 \mathrm{~m}$ de calcaires, $200 \mathrm{~m}$ de mames, cufin. dans le hant du eersant, ane alternance de bancs marneax et marno-calcaires.
Le versant concerné présente une grande homogénéité d'ensemble, du fait d'une couverture forestiere continue; it l'exception de deux champs de lavande totalisant 5 ou 6 hectares, l'ubac est entièrement boisé, et malgré une pente générale très élevée (plus de $50 \%$ ), il ne présente aucune trace de ravinement, ni mème de cours deau encaissé dans le manteau d'altération, la roche con place ou l'éboulis. Ceci autorise à penser que le ruissellement doit y être faible ; la couverture végétale est composéc essentiellement de hétres accompagnés de pins syluestres (situation climacique d'ubac). Liadret, en face, est plus sensible alt ruisiellememt ar san boisement en chénes pubescents et pins syluestres est moins dense: 


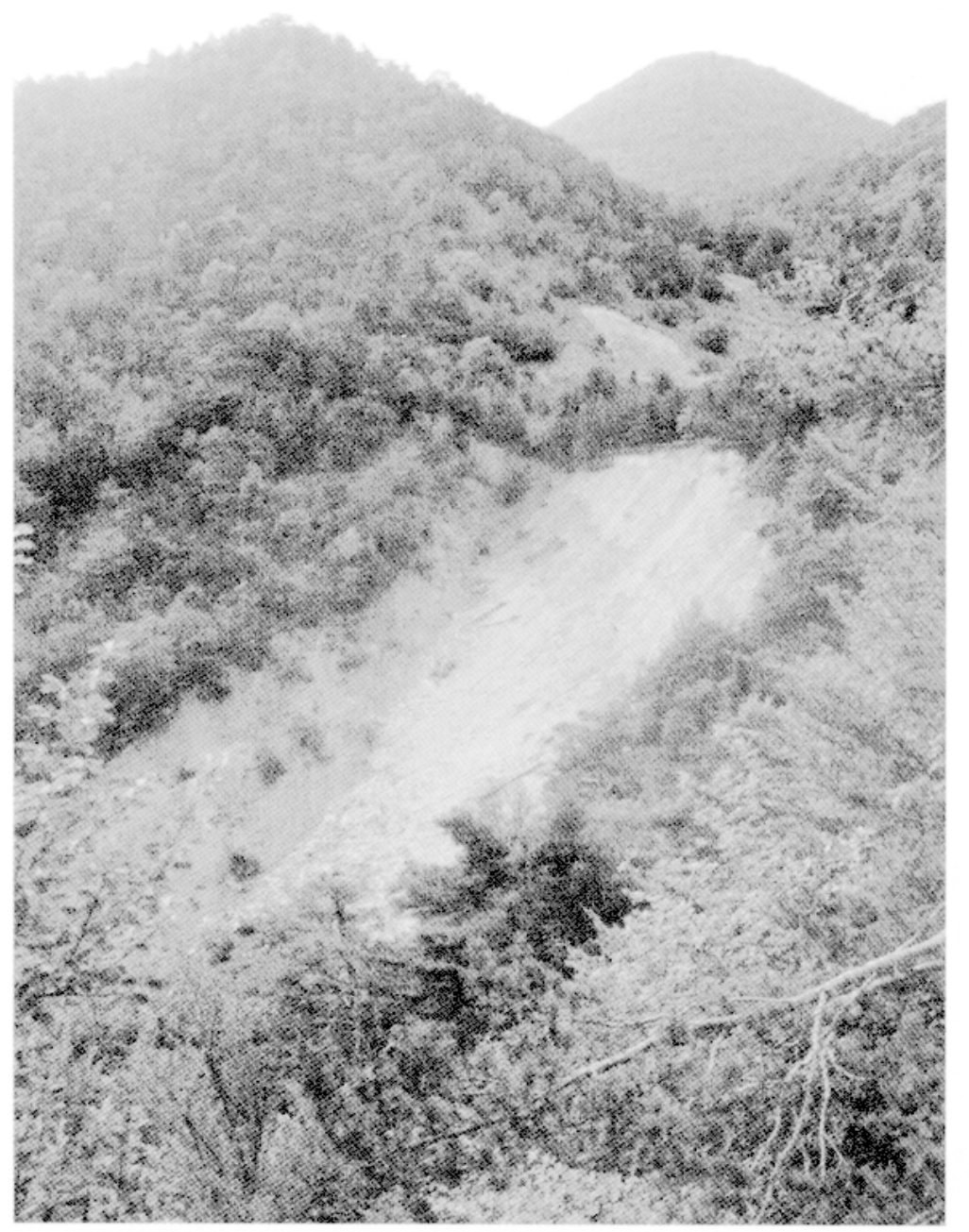

Photo 1 : La coulée boueuse de LauxMontaux (qui s'est produite en février 1978), vue d'ensemble du Nord-Ouest ; une nouvelle carriere sauvage a été ouverte durant le printemps 2001 (arriere plan)

\section{Photo 2 : La carriere révélant}

I'englacement de l'éboulis (vue du NordEst), avec son panneau signalisateur ! Au premier plan, un champ de lavande
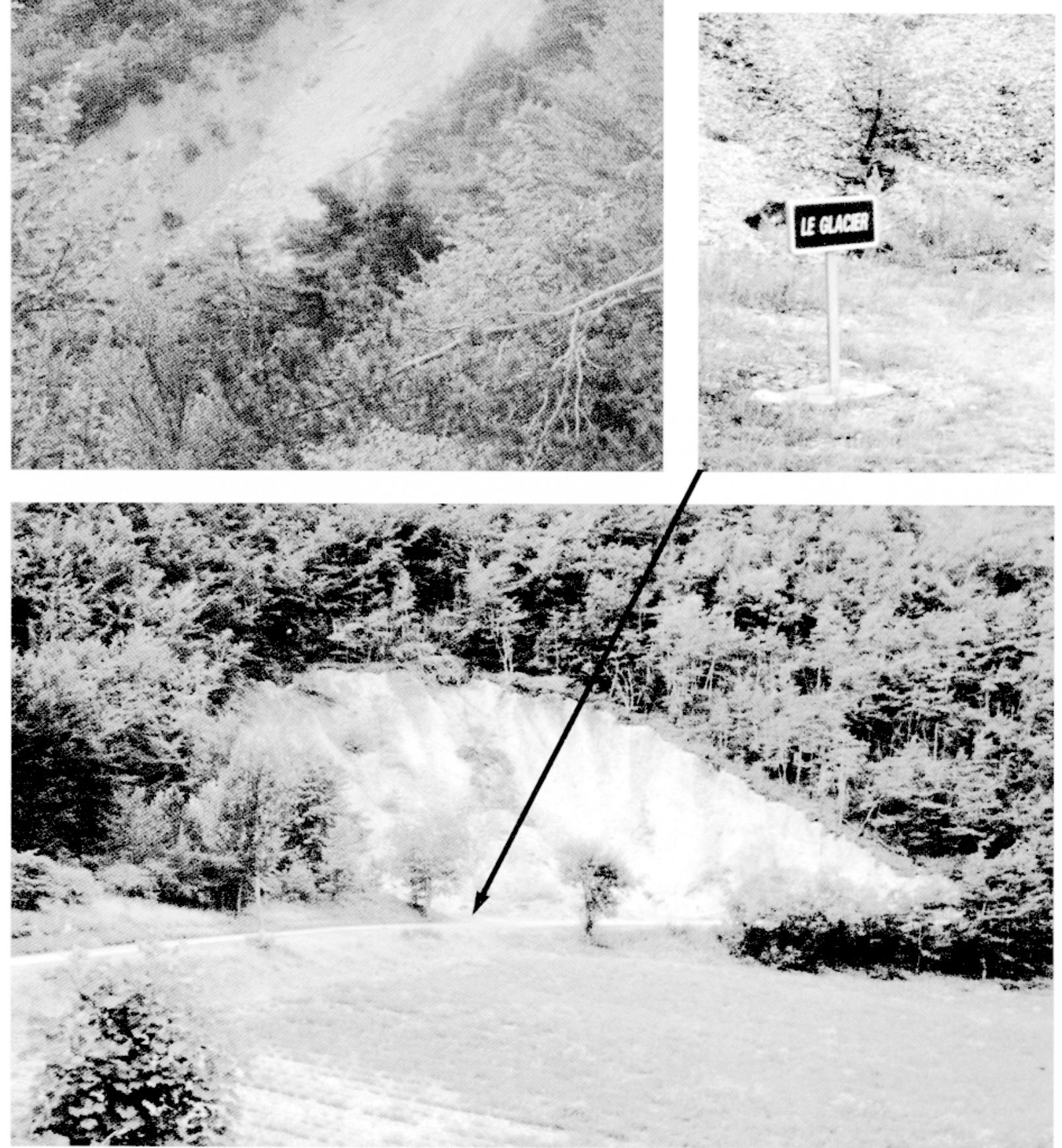
quelques ravineaux témoignent d'une plus grande agressivité de l'érosion linéatre, qui ne représente cependant en rien une menace séricuse du fait que cest un ancien parours abandonné et que la végétation y gragne d'année en année.

Cet affaissement se présente en deux parties : la partic haute, située sur l'ubac, est en fait un effondrement de l'éboulis de versant, la partie base ćtant, seule, réellement une coulée boueuse, aynt colmaté le fond du vallon sur environ 500 mètres de long et de 5 à 15 mitres d'éparisseur:

On peut supposer que la coulée a été déclenchée par un phénomène de solifluxion, lié à l'infiltration d'une grande quantité d'eau à travers l'éboulis "stabilisé"; cette ean aurait alors imprégné les marno-calcaires sous-jacents du bas du versant, leur faisant franchir leur limite de plasticité ; ceci parait confirmé par le fait que toute l'éparisseur de l'éboulis sest effondrée dans la vallée, laissant affleurer les marno-calcaires de l'Hauterivien. D'autre part, le barrage que l'effondrement a créé en travers de la vallée adjacente ne retient pas l'eau de son cours d'eatu, pourtant pérenne : on peut donc penser que les marno-calcatires nont été que peu mêlés à la

Résumé : On décrit un éboulis englacé situé dans les Baronnies. Sa localisation, relativement basse en latitude et altitude dans les Préalpes Françaises, en fait un phénomène rare et méritant une étude approfondic. Il a été le siège d'un glissement-coulée en 1978, probablement lié à la présence de la glace.

Mots-clés : glacière, éboulis, Baronnies, glissement, coulée de boue partic mobilisie, puisque ce barrage naturel est perméable.

Enfin, ce glissement, qui a ouvert une plaie blanchatre dans le versant bien boisé, faisant penser aux coulées des paysages des montagnes tropicales humides, est peut-être lié it la présence, juste au-dessus du décrochement sommital, d'un éboulis englacé, celui-ci ayant pu avoir une forte fusion it ce moment-la, laquelle aurait alimenté l'humectation de la roche en place; ceci est envisageable du fait que l'effondrement s'est produit après une grande période de précipitations, donc de relatif redoux; toutefois, la fusion devait opérer plus efficacement au redoux printanier et estival (lequel, cntrainant un dégel du sol, libere de grandes quantités d'eau en un count laps de temps); en effet, l'englacement de cet éboulis demeure tout l'été (comme en témoigne la température que l'on relieve entre les cailloux du fond de la carriere, inférieure à $l^{\circ}$ en plein été), on peut supposer que la fusion est alors très importante. Si cet englacement a joué un róle dans le déclencherment de la coulée, il faut probablement l'associer aux importantes précipitations qui l'ont précédée."

Abstract: The ice-pit scree of Laux Montaux (French Southern Alps, France). This note is about an iced scree in the Baronnies massif. Its location, at relatively low both altitude and latitude makes it a rare phenomenon. So it seems necessary to achieve thorough investigations on this site. In the same place a landslide-mud flow occurred in 1978, probably linked with the presence of ice.

Keywords: ice-pit, scree, Baronnies massif, landslide, mud flow 